Identifying the development in phase and amplitude of dipole and multipole radiation

This article has been downloaded from IOPscience. Please scroll down to see the full text article.

2012 Eur. J. Phys. 33345

(http://iopscience.iop.org/0143-0807/33/2/345)

View the table of contents for this issue, or go to the journal homepage for more

Download details:

IP Address: 92.5.124.20

The article was downloaded on 22/01/2012 at 18:07

Please note that terms and conditions apply. 


\title{
Identifying the development in phase and amplitude of dipole and multipole radiation
}

\author{
E M Rice, D S Bradshaw, K Saadi and D L Andrews ${ }^{1}$ \\ School of Chemical Sciences, University of East Anglia, Norwich NR4 7TJ, UK \\ E-mail: david.andrews@physics.org
}

Received 4 November 2011, in final form 5 December 2011

Published 19 January 2012

Online at stacks.iop.org/EJP/33/345

\begin{abstract}
The spatial variation in phase and the propagating wave-front of plane wave electromagnetic radiation are widely familiar text-book territory. In contrast, the developing amplitude and phase of radiation emitted by a dipole or multipole source generally receive less attention, despite the prevalence of these systems. There is additional complexity in such cases where, in consequence of retardation, the character and features significantly and progressively change as radiation propagates onwards, from the near-field and out towards the wavezone. Readily developed analytical representations of the electric field, cast as a function of distance from the source, provide illuminating insights into the most prominent and distinctive properties of radiant electromagnetic emission. Graphical implementations and animations of the results prove particularly instructive in revealing the spatial form and temporal evolution of the emergent electromagnetic fields.
\end{abstract}

S Online supplementary data available from stacks.iop.org/EJP/33/345/mmedia

(Some figures may appear in colour only in the online journal)

\section{Introduction}

The depiction of light as a travelling wave represents a major pillar of development in the modern understanding of optical physics, and it rightly secures an important place in most introductions to classical optics and electromagnetism. The shortcomings associated with the failure of such a representation to account for quantum phenomena demand attention soon enough, but there are other respects, less obvious but also important, in which the classical wave picture is potentially quite misleading. In particular, it might lead one to suppose that the light emanating from a source at the origin had precisely the same properties at large distances as it does close to the source, reduced intensity notwithstanding. However, it is well-known

1 Author to whom any correspondence should be addressed. 
that the character of light in the near-field (or near-zone) region, defined as a distance regime $(0, \lambda / 2 \pi)$ where $\lambda$ is the wavelength, is very different from the form of light propagating onwards, beyond the locality of the emitter. Indeed, the characteristics of propagative radiation are only acquired at distances from the source that are significantly larger than the optical wavelength.

Most optical observations involve radiation propagating in the far-field, where the distance between the source and detector antennas is much greater than the electromagnetic wavelength. However, it has emerged that near-field radiation represents an extremely useful means of conveying electromagnetic excitation over small distances, in a variety of connections. The levels of intensity required to produce a meaningful signal are much lower than for conventionally propagating light, and there is a supplementary advantage that significantly subwavelength resolution can be achieved. In the visible wavelength range, for example, where it was long anticipated that such features might be exploited in achieving enhanced resolution imaging [1], the vision has come to fruition in the techniques of near-field microscopy [2] that are now extensively used in biological applications $[3,4]$. In the same context many recent developments are associated with the local influence of surface plasmons [5], related to the special features of near-field light generated in the vicinity of nanostructured metal surfaces. Moreover, several other technologies exploiting near-field coupling operate at much longer wavelengths, namely in the radiofrequency region. While traditional radio communication is commonly conducted in the far-field, near-field communication is now increasingly being used for ticketing or payment devices, particularly through integration with mobile phones [6].

The following study aims to explore the development in amplitude and phase of light emitted by electric dipole and other multipole sources; this is suitable for introducing these and other more advanced topics in near-field optics and electromagnetic theory. The distinctive properties of light in the near-zone are identified and their progress followed as the radiation propagates onwards, from the near-field and on towards the wave-zone. Over the space of several wavelengths the functional forms of the phase and amplitude exhibit a significant change in character due to the onset of retardation. The analysis affords numerous physical insights and shows how analytical representations of the electric field, readily developed, exhibit its variation as a function of distance from the source, revealing some of the less familiar properties of radiant electromagnetic emission. It is also demonstrated how relatively straightforward graphical implementations and animations of the ensuing results can prove instructive in portraying the spatial form and temporal evolution of the emergent electromagnetic fields, clearly showing the limitations and shortcomings of a simple classical wave depiction.

\section{Background physics}

While recognizing the generality of the phenomena to be addressed, it is helpful to cultivate a physical picture by focusing on a specific wavelength regime. The transmitter-detector picture, generally applied in the microwave and radio region, is just as valid in the ultraviolet or visible electromagnetic range, and the near- and far-field forms of excitation transfer are indeed analogous in form to their radio-wave counterparts. At these shorter wavelengths, however, the dipole antennas comprise molecules, particles, ions, colour centres, etc, and the encompassing term 'chromophore' can be applied to the source and to the detector. Nearfield signal detection (here spanning typically nanoscale dimensions) is the result of energy transfer between electronic transitions in the emitter and absorber chromophores, through a coupling between the corresponding transition dipole moments [7]. The associated near-field transfer, which is highly important in many optical and photophysical applications [8-12], 
is commonly termed 'resonance energy transfer', RET; this represents the entirety of the emission and detection process. Applying the principles of quantum electrodynamics (QED) confirms that the experimentally well-characterized phenomenon of RET is the near-field asymptote of a more general coupling mechanism that applies across a full range of distances, encompassing as limits both 'radiationless' transfer (over distances $R \ll \lambda / 2 \pi$, where $\lambda$ is the optical wavelength) and 'radiative' energy transfer $(R \gg \lambda / 2 \pi)[13,14]$. Our interest focuses on the near-zone and just beyond, as the radiation covers the first few wavelengths of its path outwards from the source. A previous article has discussed the implications of quantum uncertainty within this region [15].

While the angular distributions of dipole and other multipole forms of emission are wellknown [16], it is the purpose of this paper to characterize the radial form of electromagnetic fields produced by the most common forms of multipolar emission. The aim is to identify distinctive features of the near-field, and to show how the developing amplitude and phase of radiation significantly and progressively change as radiation propagates onwards, from the near-field and out towards the wave-zone. With the use of QED methods, the retardation properties that are responsible for this development are intrinsic within the formulation. The physical picture is that of an electromagnetic transmitter (or, in terms of RET, energy donor) whose electromagnetic emission is detected by a suitably positioned and efficient probe (energy acceptor); it will be assumed that the detected signal results from resonant absorption at the frequency of the transmitted field.

The most familiar and commonplace interactions (for both emitter and detector processes) occur through electric dipolar transitions. Accordingly we begin with a brief overview of the QED derivation of the electric field, due to electric dipole emission (section 3). As a concise terminology we shall refer to this case as electric dipole-electric dipole (E1-E1) coupling. Then, since there are some instances where the donor or acceptor transition is electric dipole forbidden, on symmetry or geometric grounds, we consider in section 4 the nature of the electric field produced by electric quadrupole emission (still detected by a conventional dipolar detector, i.e. E2-E1 coupling); a detailed derivation will be presented for that case. Section 5 explores the magnetic field delivered by an electric dipole source (M1-E1 coupling), and the converse. Section 6 summarizes key aspects of the physics that can be learned from the analysis.

\section{Radial distribution of the field from an electric dipole}

As a starting point for the analysis, we shall develop a formulation of emission and detection using QED. More information on the development and detail of the methods to be employed is available in several definitive texts [17-19]; the interested reader will find a very clear exposition of the relative advantages of QED, in comparison to a semiclassical formulation, nicely set forth in a comprehensive volume by Grynberg et al [20]. To begin, the non-relativistic Hamiltonian energy of a molecular system can be presented in the following QED form:

$$
H=\sum_{\xi} H_{\mathrm{mol}}(\xi)+\sum_{\xi} H_{\mathrm{int}}(\xi)+H_{\mathrm{rad}}
$$

Here, $H_{\text {mol }}(\xi)$ and $H_{\text {rad }}$ are the respective Hamiltonians for a chromophore, $\xi$, and the radiation field; $H_{\mathrm{int}}(\xi)$ is the multipolar Hamiltonian for the interaction between the chromophore and the radiation field-which can of course be the vacuum field. For present purposes, equation (1) can be regarded as being applied to a system comprising just two material components, $\xi$ signifying the source/donor or the detector/acceptor. By inspection of this expression it is immediately apparent that, in contrast to most classical descriptions, energy transfer is not 
mediated by instantaneous coupling interactions-note the absence of any terms connecting different chromophores - but causally, through an electromagnetic field with a finite speed of propagation [21].

The term of most interest in equation (1) is the interaction term, since the light-matter interactions act as a perturbation to the total energy of the particles and the fields in isolation. We begin by considering the simplest and most frequently occurring case, in which emission occurs as the result of an electric dipole transition in the source, and the emitted radiation is detected as the result of an electric dipole-allowed transition in the detector; in consequence, what is detected is in fact the electric field delivered by the source. To determine the quantum amplitude $M^{\mathrm{e}-\mathrm{e}}$ for this E1-E1 coupling, perturbation theory is applied through the following expression:

$$
M^{\mathrm{e}-\mathrm{e}}=\sum_{r} \frac{\left\langle f\left|H_{\mathrm{int}}(\xi)\right| r\right\rangle\left\langle r\left|H_{\mathrm{int}}(\xi)\right| i\right\rangle}{\left(E_{i}-E_{r}\right)}
$$

where $|i\rangle$ and $|f\rangle$ are the initial and final system states, respectively, and $|r\rangle$ represents an intermediate state; each system state may be decomposed into its matter and radiation (Fock) states. Moreover, $E$ denotes the energy of a state denoted by the subscript. In the electric dipole approximation, $H_{\text {int }}(\xi)$ is explicitly written as

$$
H_{\text {int }}(\xi)=-\sum_{\xi} \mu(\xi) \cdot \mathbf{e}^{\perp}\left(\mathbf{R}_{\xi}\right)
$$

where the electric dipole moment operator, $\boldsymbol{\mu}(\xi)$, operates on matter states and the transverse electric field operator, $\mathbf{e}^{\perp}\left(\mathbf{R}_{\xi}\right)$, operates on radiation states (other multipole contributions to (3) are to be considered later). The explicit form of the electric field operator involves a summation over wave-vectors, $\mathbf{p}$ (magnitude $p$ ) and polarizations, $\eta$, of the radiation field, usually written in the following mode expansion:

$\mathbf{e}^{\perp}\left(\mathbf{R}_{\zeta}\right)=\mathrm{i} \sum_{\mathbf{p}, \eta}\left(\frac{\hbar c p}{2 \varepsilon_{0} V}\right)^{1 / 2}\left\{\mathbf{e}^{(\eta)}(\mathbf{p}) a^{(\eta)}(\mathbf{p}) \mathrm{e}^{\mathrm{i}\left(\mathbf{p} \cdot \mathbf{R}_{\zeta}\right)}-\overline{\mathbf{e}}^{(\eta)}(\mathbf{p}) \mathbf{a}^{\dagger(\eta)}(\mathbf{p}) \mathrm{e}^{-\mathrm{i}\left(\mathbf{p} \cdot \mathbf{R}_{\zeta}\right)}\right\}$,

where $\mathbf{e}^{(\eta)}(\mathbf{p})$ is the polarization unit vector $\left(\overline{\mathbf{e}}^{(\eta)}(\mathbf{p})\right.$ being its complex conjugate), $V$ is an arbitrary quantization volume and $a^{(\eta)}(\mathbf{p}), a^{\dagger(\eta)}(\mathbf{p})$ are respectively the annihilation and creation operators for a mode $(\mathbf{p}, \eta)$. The complicated derivation employed to determine the full expression for $M_{\mathrm{fi}}^{\mathrm{e}-\mathrm{e}}$ from equation (2), via (3) and (4), is outlined elsewhere [15]. For the purposes of this paper, the result is expressible as follows:

$$
M^{\mathrm{E} 1-E 1}=\mu_{i}^{A} V_{i j}(k, \mathbf{R}) \mu_{j}^{B}
$$

where $\boldsymbol{\mu}$ is the transition dipole moment for the component denoted by the superscript (either donor $A$ or acceptor $B$ ) and the E1-E1 coupling tensor, $V_{i j}(k, \mathbf{R})$, is cast as $[14,15]$

$$
V_{i j}(k, \mathbf{R})=\frac{\mathrm{e}^{\mathrm{i} k R}}{4 \pi \varepsilon_{0} R^{3}}\left[(1-\mathrm{i} k R)\left(\delta_{i j}-3 \hat{R}_{i} \hat{R}_{j}\right)-(k R)^{2}\left(\delta_{i j}-\hat{R}_{i} \hat{R}_{j}\right)\right] .
$$

Here, the convention of summation over repeated Cartesian indices is implemented. Furthermore, $\mathbf{R}$ is the intermolecular donor-acceptor separation vector, $\hbar c k$ is the magnitude of the energy transferred and $\mathrm{e}^{\mathrm{i} k R}$ is the phase factor (time does not feature explicitly; we introduce it later). Note that the wave-vector magnitude is not denoted by $p$ here, because $k$ is a specific value determined by the energetics of the emissive and absorptive transitions, as opposed to the summation variable in the mode expansion (4).

Continuing, the electric field emanating from the source is now determined from $E_{j}^{(E 1)}=-\mu_{i}^{A} V_{i j}(k, \mathbf{R})$, such that equation (6) gives

$$
E_{j}^{(E 1)}=\frac{\mathrm{e}^{\mathrm{i} k R}}{4 \pi \varepsilon_{0} R^{3}}\left[\left\{3(\boldsymbol{\mu} \cdot \hat{\mathbf{R}}) \hat{R}_{j}-\mu_{j}\right\}(1-\mathrm{i} k R)+\left\{\mu_{j}-(\boldsymbol{\mu} \cdot \hat{\mathbf{R}}) \hat{R}_{j}\right\} k^{2} R^{2}\right],
$$




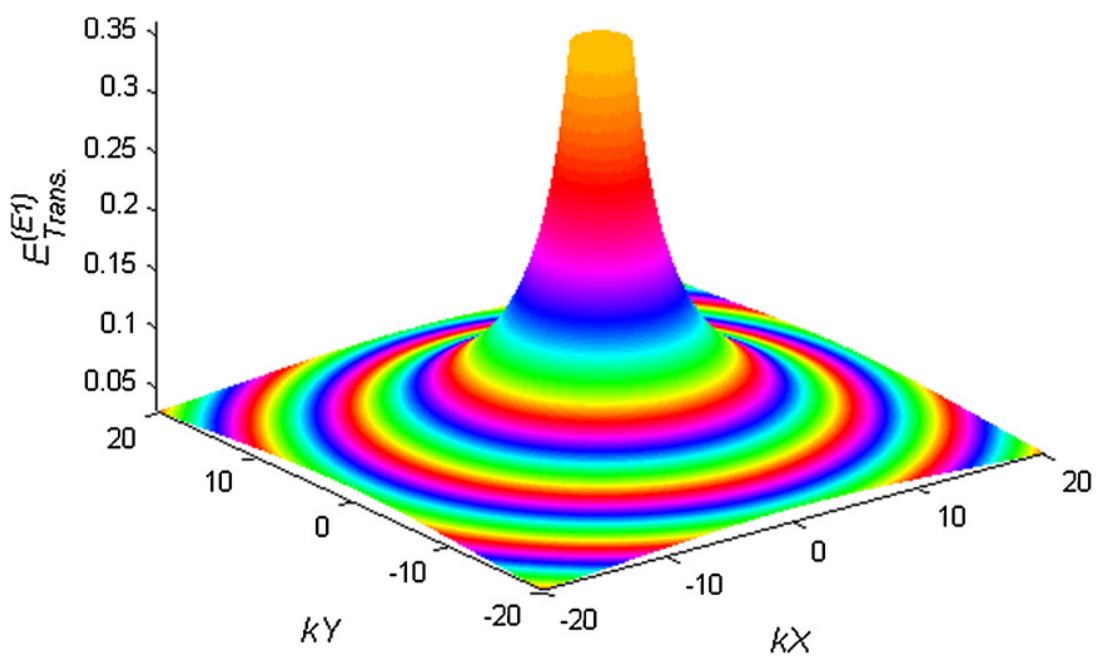

Figure 1. The transverse electric field generated by an irradiating electric dipole, as determined from equation (9). The $z$-axis represents the magnitude of the field, and the phase oscillation is depicted by the cycle of colours. The animated version showing the time evolution can be accessed by the hyperlink (see ETransvPhase.gif available at stacks.iop.org/EJP/33/345/mmedia).

dropping the redundant superscript $A$ denoting the source. Furthermore, the electric field may be split into longitudinal and transverse components (with respect to $\mathbf{R}$ ), so that $E_{\| j}^{(E 1)}=\left(E_{i}^{(E 1)} \hat{R}_{i}\right) \hat{R}_{j}$ and $E_{\perp j}^{(E 1)}=E_{j}^{(E 1)}-E_{\| j}^{(E 1)}$ represent the longitudinal and transverse components, respectively. Therefore, the explicit form of the longitudinal component of the electric field is written as

$$
E_{\| j}^{(E 1)}=\frac{\mathrm{e}^{\mathrm{i} k R}}{4 \pi \varepsilon_{0} R^{3}}\left[2(\boldsymbol{\mu} \cdot \boldsymbol{R})(1-\mathrm{i} k R) \hat{R}_{j}\right],
$$

and the transverse component

$$
E_{\perp j}^{(E 1)}=\frac{\mathrm{e}^{\mathrm{i} k R}}{4 \pi \varepsilon_{0} R^{3}}\left((\boldsymbol{\mu} \cdot \hat{\mathbf{R}}) \hat{R}_{j}-\mu_{j}\right)\left(1-\mathrm{i} k R-k^{2} R^{2}\right) .
$$

The radially cyclic phase, and the sharply declining amplitude associated with this field close to its source, are illustrated by the plot shown in figure 1, calculated for simplicity with the assumption $\boldsymbol{\mu} \perp \mathbf{R}$ (signifying a source dipole perpendicular to the plane in which the field is shown radiating outwards). In producing this plot, the magnitude of the field is determined from

$$
\left|E_{\perp}^{(E 1)}\right|=\left(E_{\perp}^{(E 1)} E_{\perp}^{(E 1) *}\right)^{\frac{1}{2}} \sim \frac{1}{R^{3}}\left(1-k^{2} R^{2}+k^{4} R^{4}\right)^{\frac{1}{2}}
$$

and the phase, $\theta$;

$$
\theta=\tan ^{-1}\left(\frac{-k R \cos k R+\sin k R-k^{2} R^{2} \sin k R}{\cos k R-k^{2} R^{2} \cos k R+k R \sin k R}\right),
$$

where the argument of the inverse tangent is the ratio of the imaginary and real parts of the transverse field - a simple exercise to prove. In figure 1, the $z$-axis represents the magnitude on an arbitrary scale, and the colours represent the phase, the latter graphically exhibiting the oscillatory behaviour of the electric field.

Before proceeding further, we note the importance of recognizing the temporal flow of radiation, responsible for the oscillation of the field at any fixed point. To this end we can 


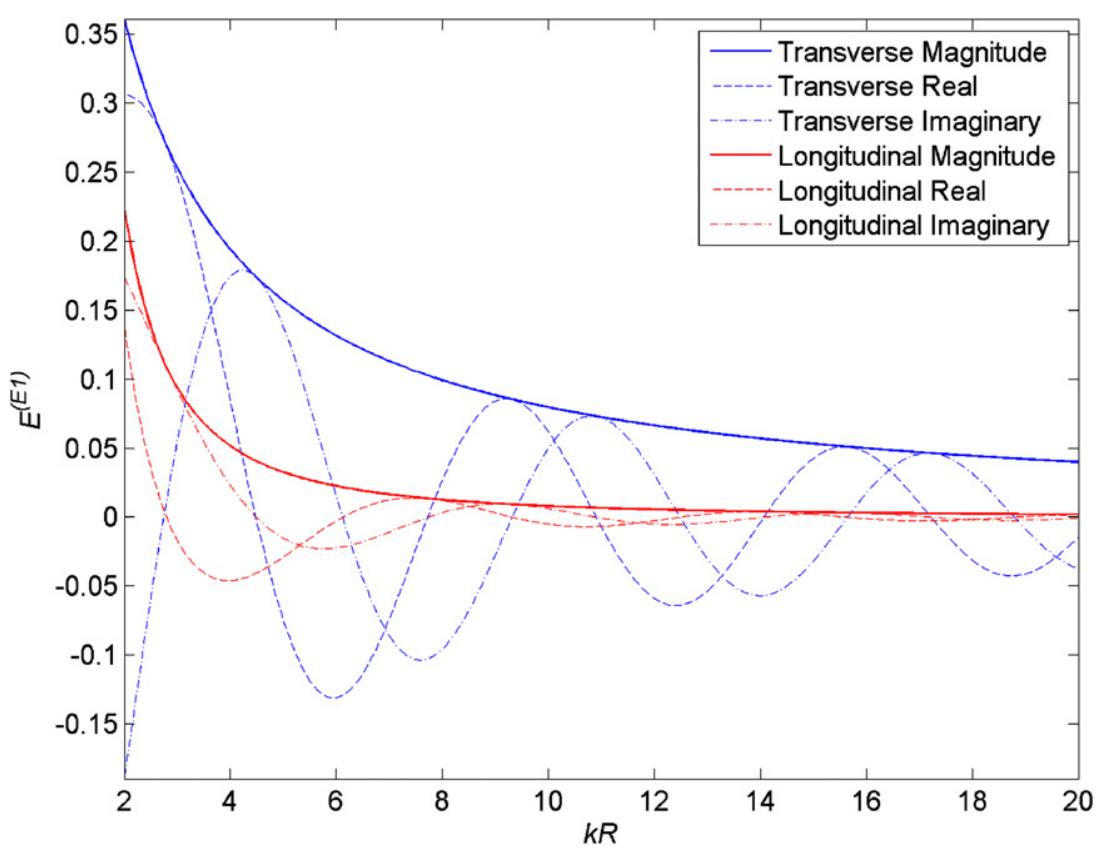

Figure 2. The magnitude of the longitudinal and transverse components (with respect to $R$ ) of an E1 dipole-emitted electric field, plotted against $k R$. The real and imaginary parts of each component are also included. The three longitudinal field curves can be indentified by their zero long-range asymptote.

introduce the time-dependent phase factor $\mathrm{e}^{-\mathrm{i} \omega t}$ driving the emission, where $\omega$ denotes the angular frequency and $t$ is the time; this develops the exponential phase factor in the electric field into the readily recognized wave form, $\mathrm{e}^{\mathrm{i} k R-\mathrm{i} \omega t}$. The associated time evolution can be visualized through animations available at stacks.iop.org/EJP/33/345/mmedia, accessible via the hyperlink attached to figure 1 .

Returning to equations (8) and (9), it is also instructive to plot the variation of transverse and longitudinal components of the electric field against $k R$; the magnitude of the real and imaginary parts of each component are separately illustrated in figure 2 . These plots lend further insights into the behaviour of the electric field in the near- and far-zone. In addition to the clear decrease in magnitude of both the transverse and longitudinal field components with distance, figure 2 clearly illustrates that the longitudinal component dominates close to the source, whilst the transverse component becomes the principal contributor as distance increases; the longitudinal component more rapidly decreases and approaches zero at larger distances. This exhibits the physical significance, in equations (8) and (9), of the fact that the transverse component contains an $R^{-1}$ leading term-which, in the long range, will dominate over the $R^{-2}$ and $R^{-3}$ terms of the longitudinal component. For additional clarity, figures 3 and 4 individually illustrate the transverse and longitudinal components, respectively, with real and imaginary parts included; once again there are animations available at stacks.iop.org/EJP/33/345/mmedia showing the time dependence, exhibiting the outward flow that cyclically interchanges the real and imaginary parts, with a phase difference of $\pi / 2$.

It is interesting to note that the wavelength of the radiating dipole field tends towards that of a free plane-wave with the same frequency, as we move away from the source. This can be seen from equation (9), for example; as $R \rightarrow \infty$, we have $E_{\perp j}^{(E 1)} \approx-\mathrm{e}^{\mathrm{i} k R} / R$, so that the zeros 


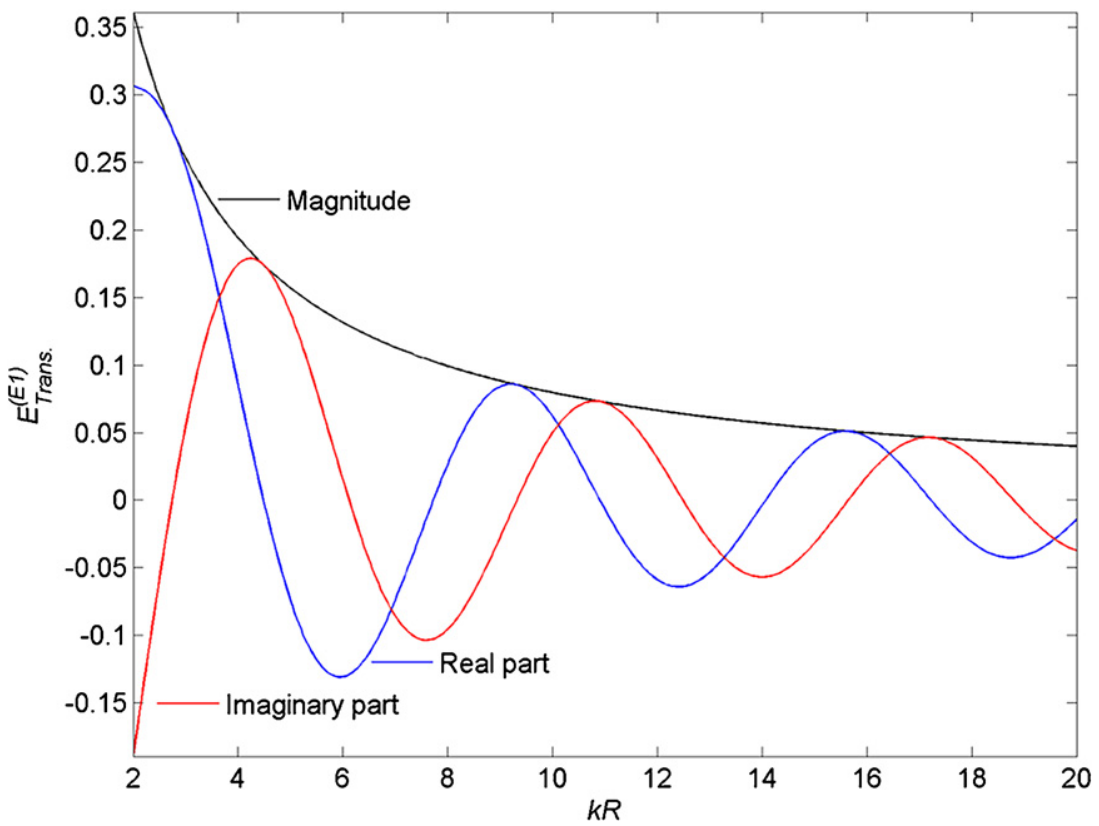

Figure 3. The magnitude of the transverse component of the E1 electric field, including its real and imaginary parts. The animated version showing the time evolution can be accessed by the hyperlink (see ETransMov.gif available at stacks.iop.org/EJP/33/345/mmedia).

Table 1. The zeros (nodes) of the real part of the electric field and the distance between two consecutive nodes (half-wavelength). The results are in units of $k R$.

\begin{tabular}{rc}
\hline \multicolumn{1}{l}{ Node } & Node distance \\
\hline 4.4817 & \\
7.7230 & 3.2413 \\
10.9033 & 3.1803 \\
14.0658 & 3.1625 \\
17.2206 & 3.1547 \\
20.3712 & 3.1506 \\
23.5194 & 3.1482 \\
26.6660 & 3.1466 \\
29.8116 & 3.1456 \\
32.9564 & 3.1448 \\
36.1006 & 3.1442 \\
39.2444 & 3.1438 \\
\hline
\end{tabular}

(nodes) of $E_{\perp j}^{(E 1)}$ are exactly those of $\mathrm{e}^{\mathrm{i} k R}$. The wavelength in units of $k R$ is exactly $2 \pi$; the interval between any two consecutive zeros is $\pi$. Close to the source at $R=0$, however, the radiation has a varying interval between successive zeros of the electric field; values can be calculated numerically by setting the real part of the field (9) equal to zero,

$$
\left(1-k^{2} R^{2}\right) \cos (k R)+k R \sin (k R)=0,
$$

which can be solved by the well-known Newton-Raphson method. Values for the first few intervals are given in table 1; for example the interval between the first two nodes is approximately 1.03 half-wavelengths. Such notable features are, indeed, measurable; they are, 


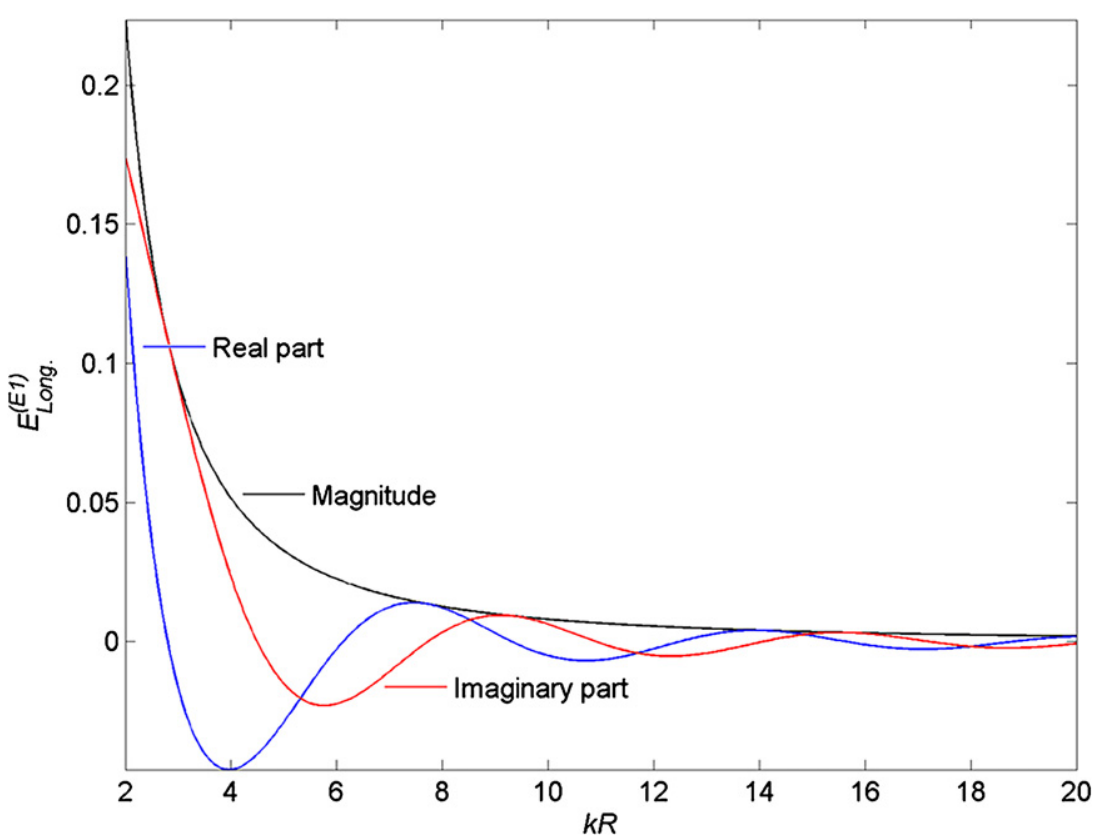

Figure 4. The magnitude of the longitudinal component of the E1 electric field, including its real and imaginary parts. The animated version showing the time evolution can be accessed by the hyperlink (see ELongMov.gif available at stacks.iop.org/EJP/33/345/mmedia).

for example, manifest in the observations of near-field electromagnetically induced forces between microscopic particles, known as optical binding [22-27].

\section{Electric field from an electric quadrupolar source}

As mentioned earlier, in cases where radiation is produced (or detected) by a chromophore transition that is electric dipole forbidden or geometrically precluded, then E1-E1 coupling no longer applies. However, subject to a suitable disposition of the relevant energy levels, radiation may nonetheless propagate from source to detector by engaging a weaker electric multipole in the dipole-forbidden transition. Accordingly, we now extend consideration to the case of electric quadrupole emission. With the linear response of a dipolar detector once again deployed to measure the emanating electric field, electric quadrupole-electric dipole coupling will dominate; we require the theory for E2-E1 coupling.

A tensor representation for E2-E1 coupling follows the same derivation procedure as previously, with the interaction Hamiltonian now written as

$$
H_{\mathrm{int}}=-\sum_{\xi}\left\{\boldsymbol{\mu}(\xi) \cdot \mathbf{e}^{\perp}\left(\mathbf{R}_{\xi}\right)+Q_{i j}(\xi) \nabla_{i} e_{j}^{\perp}\left(\mathbf{R}_{\xi}\right)\right\},
$$

where $Q_{i j}(\xi)$ is the electric quadruple operator. Generally, account should be taken of the case where the electric dipole is the transmitter and the quadrupole the detector, and vice versa. Therefore, the quantum amplitude for E2-E1 coupling is composed of two parts, $M_{\mathrm{fi}}^{\mathrm{E} 1 \mathrm{E} 2}+M_{\mathrm{fi}}^{\mathrm{E} 2-\mathrm{E} 1}$ where superscripts denote the transition multipoles for $A$ and $B$ respectively. The results emerge in the following form [28, 29]:

$$
M_{\mathrm{fi}}^{\mathrm{E} 1-\mathrm{E} 2}+M_{\mathrm{fi}}^{\mathrm{E} 2-\mathrm{E} 1}=\left\{\mu_{i}^{A} \Theta_{j k}^{B}-\Theta_{j k}^{A} \mu_{i}^{B}\right\} V_{i j k}(k, \mathbf{R}) .
$$


Here, $\Theta_{j k}$ is an electric quadrupole moment. The second term of (14) is the one of current interest, as follows from the above description. The E1-E2 coupling tensor is given by

$V_{i j k}(k, \mathbf{R})=\frac{i k^{4}}{4 \pi \varepsilon_{0}}\left[\delta_{i j} \hat{R}_{k} h_{1}^{(1)}(k R)-\frac{1}{k R}\left(\delta_{i j} \hat{R}_{k}+\delta_{i k} \hat{R}_{j}+\delta_{j k} \hat{R}_{i}\right) h_{2}^{(1)}(k R)+\hat{R}_{i} \hat{R}_{j} \hat{R}_{k} h_{3}^{(1)}(k R)\right]$,

where $h_{1}^{(1)}(k R), h_{2}^{(1)}(k R)$ and $h_{3}^{(1)}(k R)$ are Bessel functions of the following explicit form:

$$
\begin{aligned}
& h_{1}^{(1)}(k R)=-\mathrm{e}^{\mathrm{i} k R}\left(\frac{1}{k R}+\frac{i}{k^{2} R^{2}}\right), \\
& h_{2}^{(1)}(k R)=\mathrm{e}^{\mathrm{i} k R}\left(\frac{i}{k R}-\frac{3}{k^{2} R^{2}}-\frac{3 i}{k^{3} R^{3}}\right), \\
& h_{3}^{(1)}(k R)=\mathrm{e}^{\mathrm{i} k R}\left(\frac{1}{k R}+\frac{2 i}{k^{2} R^{2}}-\frac{15}{k^{3} R^{3}}-\frac{15 i}{k^{4} R^{4}}\right) .
\end{aligned}
$$

Equation (15) is related to the quadrupole field by the expression $E_{i}^{(E 2)}=\Theta_{j k}^{A} V_{i j k}(k, \mathbf{R})$, so that

$E_{i}^{(E 2)}=\frac{i k^{4}}{4 \pi \varepsilon_{0}}\left[\Theta_{i k} \hat{R}_{k} h_{1}^{(1)}(k R)-\frac{1}{k R}\left(\Theta_{i k} \hat{R}_{k}+\Theta_{j i} \hat{R}_{j}+\Theta_{j j} \hat{R}_{i}\right) h_{2}^{(1)}(k R)+\Theta_{j k} \hat{R}_{i} \hat{R}_{j} \hat{R}_{k} h_{3}^{(1)}(k R)\right]$.

Since the dummy indices are arbitrary and the transition quadrupole moment is traceless, this simplifies to

$E_{i}^{(E 2)}=\frac{i k^{4}}{4 \pi \varepsilon_{0}}\left[\Theta_{i k} \hat{R}_{k}\left(h_{1}^{(1)}(k R)-\frac{2}{k R} h_{2}^{(1)}(k R)\right)+\Theta_{j k} \hat{R}_{i} \hat{R}_{j} \hat{R}_{k} h_{3}^{(1)}(k R)\right]$.

As previously, for the electric dipole field, the longitudinal component of the quadrupole field is given by $E_{\| i}^{(E 2)}=\left(E_{i}^{(E 2)} \hat{R}_{l}\right) \hat{R}_{i}$. Thus, from equation (18) we obtain

$E_{\| i}^{(E 2)}=\frac{i k^{4}}{4 \pi \varepsilon_{0}}\left[\Theta_{i k} \hat{R}_{k} \hat{R}_{i} \hat{R}_{l}\left(h_{1}^{(1)}(k R)-\frac{2}{k R} h_{2}^{(1)}(k R)\right)+\Theta_{j k} \hat{R}_{l} \hat{R}_{j} \hat{R}_{k} h_{3}^{(1)}(k R)\right]$,

which, since the dummy indices can be relabelled without changing the expression, simplifies to

$$
E_{\| i}^{(E 2)}=\frac{i k^{4}}{4 \pi \varepsilon_{0}} \Theta_{j k} \hat{R}_{i} \hat{R}_{j} \hat{R}_{k}\left\{h_{1}^{(1)}(k R)-\frac{2}{k R} h_{2}^{(1)}(k R)+h_{3}^{(1)}(k R)\right\} .
$$

The transverse component is determined from $Q_{i}^{\perp}=Q_{i}-Q_{i}^{I I}$, so that

$$
E_{\perp i}^{(E 2)}=\frac{i k^{4}}{4 \pi \varepsilon_{0}} \Theta_{i k} \hat{R}_{k}\left(1-\hat{R}_{i} \hat{R}_{j}\right)\left\{h_{1}^{(1)}(k R)-\frac{2}{k R} h_{2}^{(1)}(k R)\right\} .
$$

Substituting in the Bessel functions, and simplifying, equations (20) and (21) become

$$
\begin{aligned}
& E_{\| i}^{(E 2)}=\frac{k^{4} \mathrm{e}^{\mathrm{i} k R}}{4 \pi \varepsilon_{0}} \Theta_{j k} \hat{R}_{i} \hat{R}_{j} \hat{R}_{k}\left(\frac{1}{k^{2} R^{2}}-\frac{9 i}{k^{3} R^{3}}+\frac{9}{k^{4} R^{4}}\right), \\
& E_{\perp i}^{(E 2)}=\frac{k^{4} \mathrm{e}^{\mathrm{i} k R}}{4 \pi \varepsilon_{0}} \Theta_{j k} \hat{R}_{k}\left(\hat{R}_{i} \hat{R}_{j}-1\right)\left(\frac{i}{k R}-\frac{3}{k^{2} R^{2}}-\frac{6 i}{k^{3} R^{3}}+\frac{6}{k^{4} R^{4}}\right) .
\end{aligned}
$$

The magnitudes of both the transverse and longitudinal components of this field, produced by a quadrupolar source, are illustrated in figure 5 . The figure shows that both components of the quadrupole field decrease much more quickly with distance from the transmitter, than in the E1-E1 case. This is due to the presence in equations (20) and (21) of an $R^{-4}$ term, which does not appear in the expression for E1-E1 coupling; as a result, the effective range 


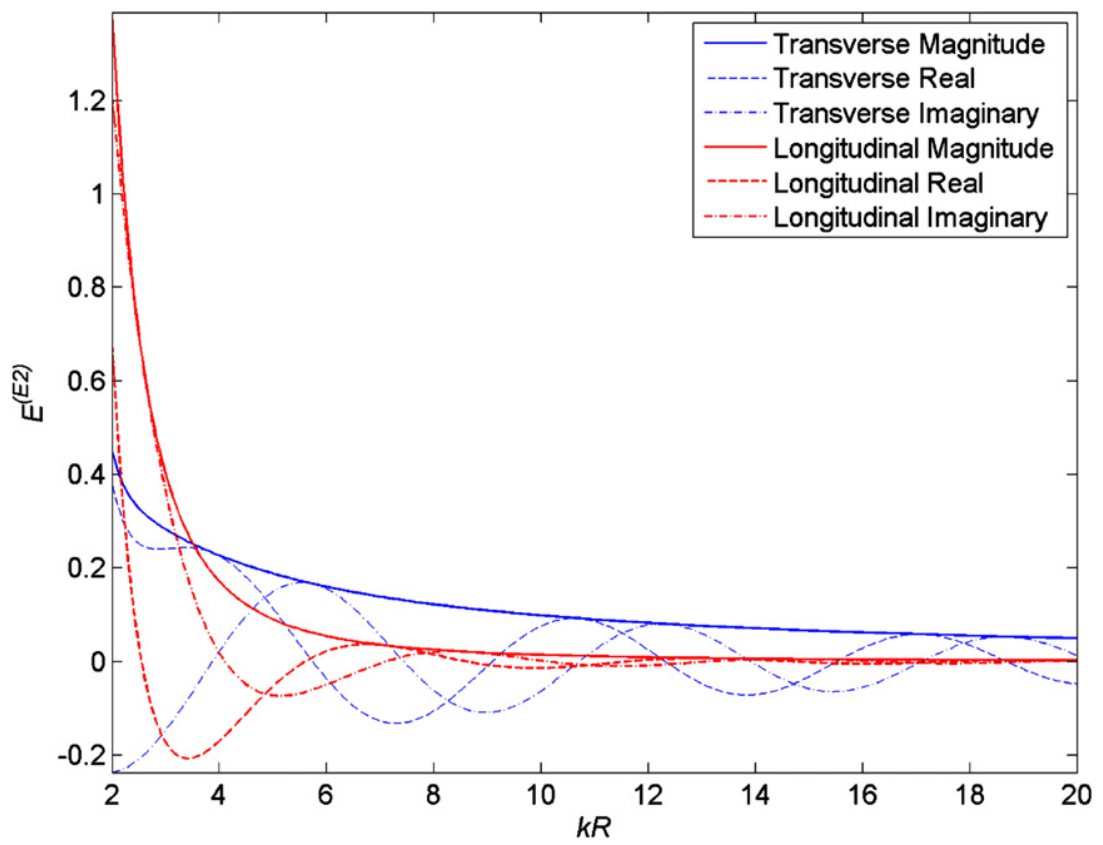

Figure 5. The magnitude of the transverse and longitudinal components of the E2 electric quadrupole field, including its real and imaginary parts. The three longitudinal field curves can be identified by their zero long-range asymptote.

of the electric quadrupole field is small compared to that of the electric dipole. The graph also shows that the longitudinal component dominates in the near-field, and both components tend to zero in the far-field. Comparison with the earlier graphs again shows the characteristic oscillatory behaviour of the real and imaginary parts of each component, but now much more greatly damped. Figures 6 and 7, which show the separate components for clarity, can again be regarded as snapshots of a time-dependent evolution, explicitly exhibited in animations available at stacks.iop.org/EJP/33/345/mmedia accessible from the embedded hyperlinks.

\section{Electric field from a magnetic dipole source, and vice versa}

For the case of a magnetic dipole source, again with the use of an electric dipolar detector for the electromagnetic field so produced, we require the development of theory for M1-E1 coupling. The interaction Hamiltonian is now

$$
H_{\text {int }}=-\sum_{\xi}\left\{\mu(\xi) \cdot \mathbf{e}^{\perp}\left(\mathbf{R}_{\xi}\right)+\mathbf{m}(\xi) \cdot \mathbf{b}\left(\mathbf{R}_{\xi}\right)\right\},
$$

which exhibits both the magnetic dipole operator, $\mathbf{m}(\xi)$, and the magnetic field operator, $\mathbf{b}\left(\mathbf{R}_{\xi}\right)$. The latter operator may be cast as a mode expansion:

$\mathbf{b}\left(\mathbf{R}_{\xi}\right)=\mathrm{i} \sum_{\mathbf{p}, \lambda}\left(\frac{\hbar k}{2 \varepsilon_{0} c V}\right)^{\frac{1}{2}}\left\{\mathbf{b}^{(\lambda)}(\mathbf{p}) a^{(\lambda)}(\mathbf{p}) \mathrm{e}^{\mathrm{i} \mathbf{p} \cdot \mathbf{R}_{\xi}}-\overline{\mathbf{b}}^{(\lambda)}(\mathbf{p}) a^{\dagger(\lambda)}(\mathbf{p}) \mathrm{e}^{-\mathrm{i} \mathbf{p} \cdot \mathbf{R}_{\xi}}\right\}$, 


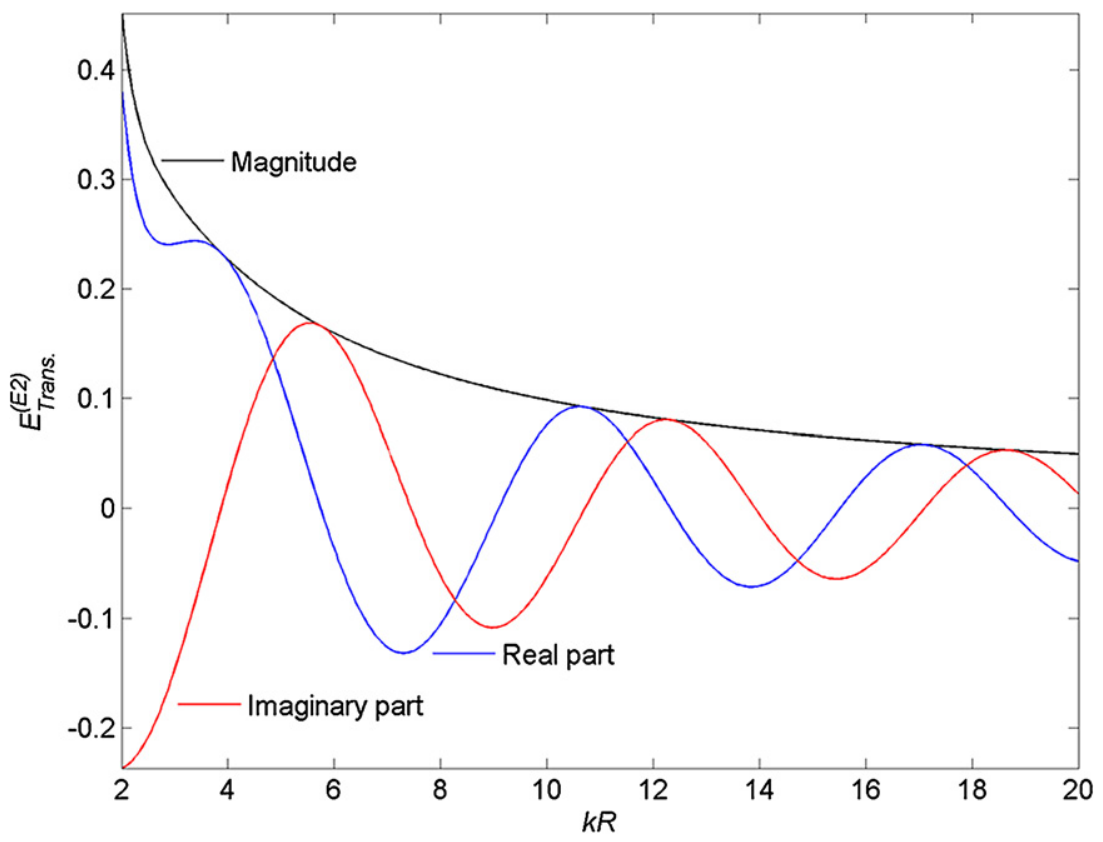

Figure 6. The magnitude of the transverse component of the E2 quadrupole field, including its real and imaginary parts. The animated version showing the time evolution can be accessed by the hyperlink (see QTransMov.gif available at stacks.iop.org/EJP/33/345/mmedia).

where $\mathbf{b}^{(\lambda)}(\mathbf{p})=\hat{\mathbf{p}} \times \mathbf{e}^{(\lambda)}(\mathbf{p})$ is the magnetic polarization vector in the direction of the magnetic field vector and $\overline{\mathbf{b}}^{(\lambda)}(\mathbf{p})$ is its complex conjugate. Once again, the quantum amplitude for M1E1 coupling comprises two parts, $M_{\mathrm{fi}}^{\mathrm{E} 1-\mathrm{M} 1}+M_{\mathrm{fi}}^{\mathrm{M} 1-\mathrm{E} 1}$, and is determined as $[10,12]$

$$
M_{\mathrm{fi}}^{\mathrm{E} 1-\mathrm{M} 1}+M_{\mathrm{fi}}^{\mathrm{M} 1-\mathrm{E} 1}=\left(\mu_{i}^{A} m_{j}^{B}+m_{j}^{A} \mu_{i}^{B}\right) U_{i j}(k, \mathbf{R}),
$$

where $\mathbf{m}$ denotes a magnetic transition dipole moment. The second term of (26) reflects dipolar detection of an electric field $E_{i}^{M 1}=-m_{j}^{A} U_{i j}(k, \mathbf{R})$, produced by magnetic dipole emission. Conversely, the first term of (26) represents the dipolar detection of a magnetic field, as would be produced by electric dipole emission. Both are of exactly similar form and engage the same coupling tensor, given by

$$
U_{i j}(k, \mathbf{R})=\frac{\mathrm{e}^{\mathrm{i} k R}}{4 \pi \varepsilon_{0}} \varepsilon_{i j k} \frac{\hat{R}_{k}}{R^{3}}\left(-\mathrm{i} k R-k^{2} R^{2}\right) .
$$

Thus the magnetic field resulting from electric dipole emission is related to the coupling tensor through $B_{j}^{E 1}=-\mu_{i}^{A} U_{i j}(k, \mathbf{R})$, so that

$$
B_{j}^{E 1}=\frac{\mathrm{e}^{\mathrm{i} k R}}{4 \pi \varepsilon_{0} R^{3}} \mu_{i} \hat{R}_{k} \varepsilon_{i j k}\left(\mathrm{i} k R+k^{2} R^{2}\right),
$$

again in agreement with [16]. By contrast to the electric field in the E1-E1 and E2-E1 cases, equations (7) and (18), this magnetic field is entirely transverse with respect to $\mathbf{R}$. The transverse component of the magnetic field has a similar graphical form to its electric counterpart (figure 8). Once again, the magnitude of the field decreases with increasing distance from the source, the real and imaginary parts oscillating out of phase by $\pi / 2$; however the near-field is dominated by the term with an inverse square dependence on $R$. It is noteworthy 


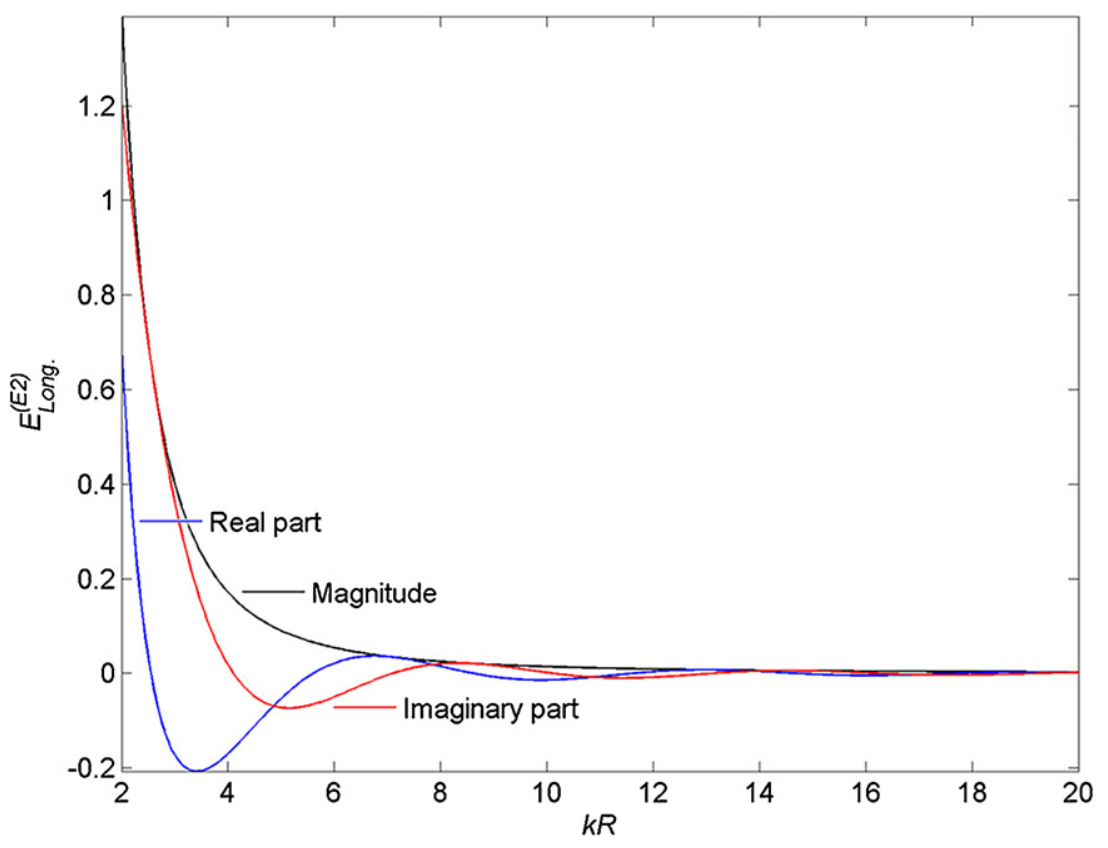

Figure 7. The magnitude of the longitudinal component of the E2 quadrupole field, including its real and imaginary parts. The animated version showing the time evolution can be accessed by the hyperlink (see QLongMov.gif available at stacks.iop.org/EJP/33/345/mmedia).

that the electric field resulting from magnetic dipole emission simply involves the replacement of $\boldsymbol{\mu}$ with $\mathbf{m}$ in equation (28); the form of the associated plot will be identical to figure 5 . For completeness, and in passing, we note that M1-M1 coupling takes precisely the same form as the E1-E1 coupling discussed earlier.

\section{Discussion}

The above analysis amply illustrates the significance of the distance regime in which the electromagnetic fields of light are measured, relative to their source, over the first few wavelengths of optical propagation. We have previously exhibited the physical implications of quantum uncertainty in this regime [15], showing its manifestation in the derivations of the multipole coupling tensors. Here we have focused on the developing amplitude and phase of the emitted radiation, their interplay even proving to produce an effective variation in wavelength close to the source. These matters are not only of fundamental interest; the subject area also has great practical importance in connection with ongoing developments at the forefront of nanophotonics, where work is progressing towards the creation of ultra-compact optical antennas [30]. Before concluding, we note here a few other lessons that can be learned from this discourse.

First, it has become apparent that the form of tensor coupling between any given electric or magnetic multipolar transitions, involved in emission and detection, is invariant with respect to interchange of those roles. This is made evident because we have taken care to include a full representation of the energy transfer process, including detection, in an RET development. Hence it becomes obvious that the magnetic field emitted by a regular electric dipolar source 


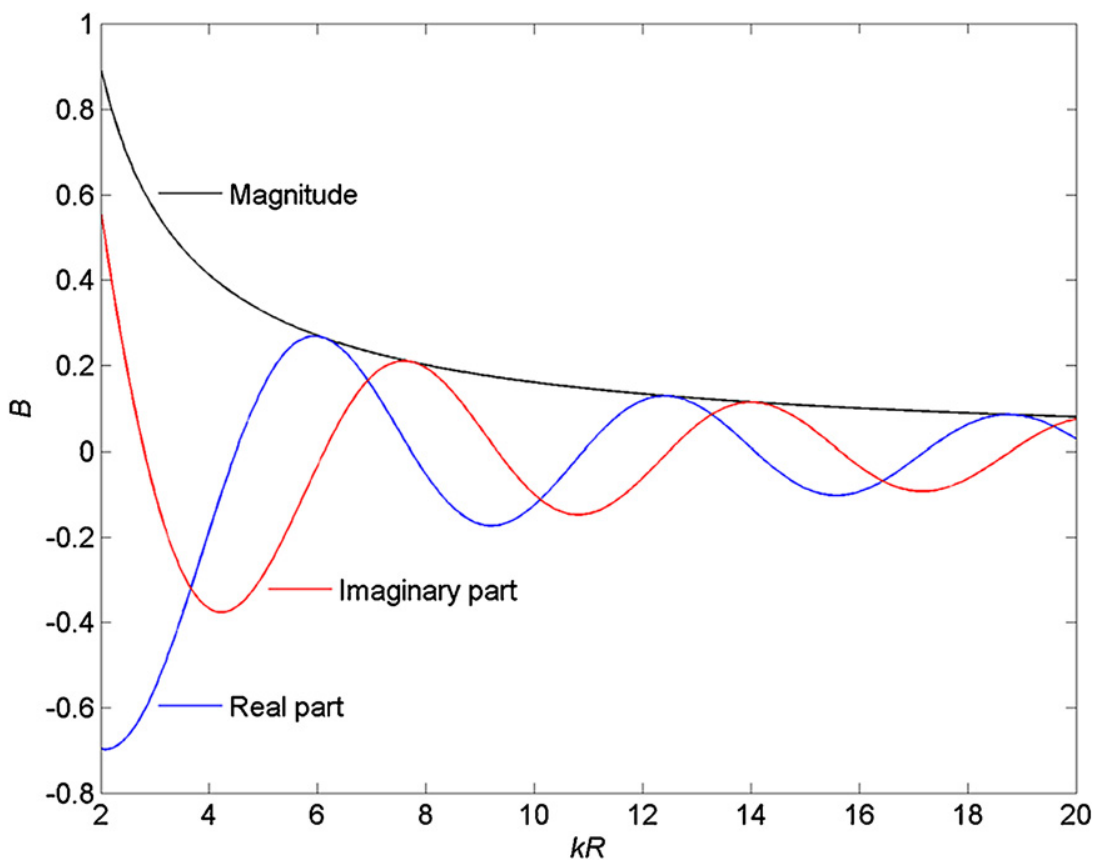

Figure 8. The magnitude of a magnetic field deriving from an electric dipole, including its real and imaginary parts.

is exactly similar, in the radial dependence of its magnitude and phase, to that of an electric field emitted by a magnetic dipole. In this particular case however-in marked contrast to the components of the electric field in E1-E1 coupling, equations (8) and (9), and E2-E1 coupling, equations (22) and (23) — there is only a transverse field (28). Moreover this disappears when $k=0$. The latter observation is not surprising; it registers the fact that static electric dipoles do not interact with static magnetic dipoles. Nonetheless, the difference in behaviour when $k \neq 0$ flags the fact that this preclusion of coupling between electric and magnetic dipoles no longer applies when those concerned are transition dipoles, and energy flows between the particles thus coupled. One feature that all of these forms of coupling have in common is that in their long-range asymptotes, the magnitude of each field runs with $R^{-1}$, as befits the inverse square law in the associated electromagnetic intensities.

We conclude by observing that this analysis affords obvious opportunities to write code for static and animated graphical depictions whose visualizations further assist interpretation. The tensor algebra is straightforward and a wide range of physical insights is afforded by the analysis. In principle, experimental realizations are also possible; as has been noted, optical binding experiments afford opportunities to mechanically register the precisely damped oscillations in field dependence that characterize the exhibited graphics.

\section{References}

[1] Synge E H 1928 A suggested method for extending the microscopic resolution into the ultramicroscopic region Phil. Mag. 6 356-62

[2] Betzig E and Trautman J K 1992 Near-field optics: microscopy, spectroscopy, and surface modification beyond the diffraction limit Science 257 189-95 
[3] Lewis A, Taha H, Strinkovski A, Manevitch A, Khatchatouriants A, Dekhter R and Ammann E 2003 Near-field optics: from subwavelength illumination to nanometric shadowing Nature Biotechnol. 21 1378-86

[4] Dickenson N E, Armendariz K P, Huckabay H A, Livanec P W and Dunn R C 2010 Near-field scanning optical microscopy: a tool for nanometric exploration of biological membranes Anal. Bioanal. Chem. 396 31-43

[5] Novotny L 2011 From near-field optics to optical antennas Phys. Today 64 47-52

[6] Want R 2011 Near-field communication IEEE Pervasive Comput. 10 4-7

[7] Andrews D L and Bradshaw D S 2009 Encyclopedia of Applied Spectroscopy ed D L Andrews (Weinheim: Wiley) chapter 15

[8] Shankar K, Feng X and Grimes C A 2009 Enhanced harvesting of red photons in nanowire solar cells: evidence of resonance energy transfer ACS Nano 3 788-94

[9] Mor G K, Basham J, Paulose M, Kim S, Varghese O K, Vaish A, Yoriya S and Grimes C A 2010 High-efficiency Förster resonance energy transfer in solid-state dye sensitized solar cells Nano Lett. 10 2387-94

[10] Laptenok S P, Borst J W, Mullen K M, van Stokkum I H M, Visser A J W G and van Amerongen H 2010 Global analysis of Förster resonance energy transfer in live cells measured by fluorescence lifetime imaging microscopy exploiting the rise time of acceptor fluorescence Phys. Chem. Chem. Phys. 12 $7593-602$

[11] Deng S, Chen J, Huang Q, Fan C and Cheng Y 2010 Saturated Förster resonance energy transfer microscopy with a stimulated emission depletion beam: a pathway toward single-molecule resolution in far-field bioimaging Opt. Lett. 35 3862-4

[12] Rusanov A L and Savitsky A P 2011 Fluorescence resonance energy transfer between fluorescent proteins as powerful toolkits for in vivo studies Laser Phys. Lett. 8 91-102

[13] Andrews DL 1989 A unified theory of radiative and radiationless molecular energy transfer Chem. Phys. 135 195-201

[14] Daniels G J, Jenkins R D, Bradshaw D S and Andrews D L 2003 Resonance energy transfer: the unified theory revisited J. Chem. Phys. $1192264-74$

[15] Andrews DL and Bradshaw D S 2004 Virtual photons, dipole fields and energy transfer: a quantum electrodynamical approach Eur. J. Phys. 25 845-58

[16] Jackson J D 1999 Classical Electrodynamics 3rd edn (New York: Wiley) chapter 9

[17] Cohen-Tannoudji C, Dupont-Roc J and Grynberg G 1989 Photons and Atoms. Introduction to Quantum Electrodynamics (New York: Wiley)

[18] Craig D P and Thirunamachandran T 1998 Molecular Quantum Electrodynamics. An Introduction to RadiationMolecule Interactions (New York: Dover)

[19] Salam A 2010 Molecular Quantum Electrodynamics. Long-Range Intermolecular Interactions (Hoboken, NJ: Wiley)

[20] Grynberg G, Aspect A and Fabre C 2010 Introduction to Quantum Optics. From the Semi-Classical Approach to Quantized Light (Cambridge: Cambridge University Press)

[21] Andrews D L 2008 Mechanistic principles and applications of resonance energy transfer Can. J. Chem. $86855-70$

[22] Burns M M, Fournier J-M and Golovchenko J A 1989 Optical binding Phys. Rev. Lett. $631233-6$

[23] Burns M M, Fournier J-M and Golovchenko J A 1990 Optical matter: crystallization and binding in intense optical fields Science 249 749-54

[24] McGloin D, Carruthers A E, Dholakia K and Wright E M 2004 Optically bound microscopic particles in one dimension Phys. Rev. E 69021403

[25] Rodríguez J, Dávila Romero L C and Andrews D L 2007 Optically induced potential energy landscapes J. Nanophotonics 1019503

[26] Andrews D L and Dávila Romero L C 2009 Mechanisms for optical binding Proc. SPIE $740074001 \mathrm{H}$

[27] Čižmár T, Dávila Romero L C, Dholakia K and Andrews D L 2010 Multiple optical trapping and binding: new routes to self-assembly J. Phys. B: At. Mol. Opt. Phys. 43102001

[28] Scholes G D and Andrews D L 1997 Damping and higher multipole effects in the quantum electrodynamical model for electronic energy transfer in the condensed phase J. Chem. Phys. 107 5374-84

[29] Salam A 2005 A general formula for the rate of resonant transfer of energy between two electric multipole moments of arbitrary order using molecular quantum electrodynamics J. Chem. Phys. 122044112

[30] Rolly B, Stout B, Bidault S and Bonod N 2011 Crucial role of the emitter-particle distance on the directivity of optical antennas Opt. Lett. 36 3368-70 